\title{
Cognitive load management in mobile learning systems: principles and theories
}

\author{
Brita Curum $^{1}$ - Kavi Kumar Khedo ${ }^{1}$
}

Received: 23 April 2020 / Revised: 30 July 2020 / Accepted: 31 July 2020 /

Published online: 10 August 2020

(C) Beijing Normal University 2020

\begin{abstract}
With the widespread adoption of mobile technologies, mobile-assisted learning is gaining lots of momentum. This new learning paradigm promotes education across different contexts, which is a key factor that contributes to enhancing learning irrespective of the conditions and location of the learner. Therefore, it creates an authentic learning setting whereby students can make meaningful connections to the real world while learning takes place. Previous research works in the field of mobile learning showed that improper design of learning elements is still present in mobile systems and consequently results in poor dynamic content adaptation. Some attempts to adapt learning contents with appropriate instructional design principles are conducted, but with moderate exploitation of smart technological assets in mobile learning systems and limited pedagogical reflections and cognitive factors. In this paper, a learning efficiency model chart is derived using important learning factors that can be considered to enhance mobile learning experiences. Some popular learning theories are analysed and compared with the proposed learning efficiency model chart. This investigation is considered to significantly reduce complexities that exist in mobile learning platforms and promote an enhanced mobile learning experience.
\end{abstract}

Keywords Mobile learning - Adaptation strategies · Cognitive load theory · Instructional design $\cdot$ Cognitive comparative analysis

Brita Curum

brita_curum@hotmail.com

Kavi Kumar Khedo

k.khedo@uom.ac.mu

1 Faculty of Information, Communication and Digital Technologies, University of Mauritius, Réduit, Mauritius 


\section{Introduction}

The omnipresent characteristic of mobile devices allows learning to take place beyond traditional spaces. With this highly adopted learning trend, studies pointed out that too much information or improper design of multimedia contents cause unnecessary cognitive load (Salonen et al. 2005; Efklides 2008) and eventually harm the learning effectiveness. It is widely agreed that mobile learning as a tool for learning needs further development in terms of pedagogy and instructional design techniques to attain higher potential (Wang and Shen 2012; Olmos et al. 2018; Feldon et al. 2019). Moreover, Zafar and Hasan (2014) pointed out that limited content adaptation using the learners' context information hinders the learning experience and makes it more difficult to learn. This urges an analysis of the cognitive load on learners based on the amount of information flow they are presented with. The human cognitive architecture which is composed of the working memory can handle only a very limited number of interacting elements (Baddeley and Hitch 1974). Thus, a lack of good design of learning elements and instructional strategies is prone to overload the working memory of learners. As a result, to bring forward learning effectiveness in mobile learning, the learning contents presented needs to be re-adapted and re-structured based on appropriate cognitive theories.

As learning materials are being designed to appropriately fit mobile screens and simultaneously allowing good knowledge transfer on complex learning topics, the importance of instructional design principles together with cognitive load theories cannot be disregarded. In this paper, a review for cognitive load and instructional design theories relevant to mobile learning is carried out. Different learning theories and instructional design principles supporting both formal and informal learning in mobile learning are explored. Sect. Mobile learning introduces the concept of mobile learning. Sect. Cognitive load principles for mobile learning describes the Cognitive Load Theories for Mobile Learning and evaluates the different types of cognitive load for mobile learning. Section 5 describes some impacts of cognitive load on mobile learning. Sect. The efficiency of cognitive load theories for mobile learning provides a comparative analysis of the different Cognitive Load Theories followed by Sect. Instructional design principles for managing cognitive load in mobile learning which defines some of the most used instructional design principles in mobile learning. Finally, Sect. Discussions discusses the observations made concerning Cognitive Load Theories and Sect. Conclusions concludes the paper.

\section{Research methodology}

As mobile learning is exponentially playing an important role in education these days, the potential of cognitive load theories cannot be disregarded. Therefore, to retain the attention and motivation of mobile learners during a learning process, 
proper cognitive load theories should be considered. A research methodology was derived to investigate the high impact factors related to cognitive load theories on mobile learning. There has been considerable research on cognitive load theories but a consolidation (survey) of those theories for mobile learning is missing in existing literature. From a practical perspective, this will help mobile learning platform developers to have good visibility of the cognitive mechanism for mobile learning and allow efficient interventions.

This review on the cognitive load theories in mobile learning has been performed using the Kitchenham method (Kitchenham et al. 2009) whereby important research questions have been set and narrowed down through the PRISMA (Preferred Reporting Items for Systematic Reviews and Meta-Analyses) technique where some important pieces of evidence are put forward for the evaluation of this study (Moher et al. 2015). The investigation funnel is organized in the following steps: planning the review, conducting the review, the inclusion and exclusion criteria and finally the search phase where the review is documented.

\section{Planning the review}

Cognitive load theory is gaining popularity in context-aware teaching systems, and this paper highlights some important related work and its achievements in mobile learning. A comparison schema for the integration of cognitive load theory is then derived and analysed for mobile learning. Instructional design principles for the management of cognitive load in mobile learning are also explored.

\section{Research questions}

The main research questions addressed in this paper are as follows:

RQ1 What are the most important learning theories that apply to mobile learning?

In this study, we will investigate the three main theories of learning (behaviourism, cognitivism and constructivism) and how they can be useful to enhance the learning pattern of an individual using a mobile phone.

RQ2 What are the important instructional design principles in mobile learning and how do they promote better learning?

Mobile phones are by default smaller in size. Therefore, it is imperative to consider instructional design principles to deliver learning materials that fit and ultimately showcase non-complex data, as a measure to maximize learning in such a platform.

RQ3 How cognitive load and pedagogical factors can be managed in mobile learning systems? 
It is observed that most of the cognitive load theories use factors such as the motivation of the learner and the learners' individual capabilities to reformulate learning elements. Emotional traits are less impactful for cognitive assessment in mobile learning. The behaviour of the learner needs to be focused on since the decisions of the learner could be dependent on his mood during a mobile learning process.

\section{Conducting the review}

\section{The searching process}

The steps followed for the search process is as follows: identify the most recent and relevant cognitive load theories, learning effects and instructional design principles to evaluate their relations with mobile learning platforms using Google Scholar.

\section{Inclusion and exclusion criteria}

The inclusion and exclusion criteria were defined as follows:

\section{Inclusion}

- Most recent studies were considered (last 5 years).

- Keywords such as cognitive load, adaptation strategies, cognitive load theories, mobile learning, learning effects, game-based learning, fun learning, self-regulated learning, learning theories and instructional design principles were queried.

- Papers related to cognitive load theories, cognitive comparative analysis and instructional-designed principles in mobile learning platforms.

\section{Exclusion}

- Cognitive load theory articles for general or classroom-based learning.

- Cyber security issues related to learning flow.

- Bio-medical impact in a virtual remote environment.

- Chinese language-related articles.

Based on the above criteria, data were extracted for quality assessment.

\section{Search}

There were 60 articles related to the keyword search in Google Scholar. Recently published papers were filtered against full-text review with quality assessment and cognitive comparative analysis. This returned 55 articles. The records were then screened manually with both the inclusion and exclusion criteria and 43 relevant articles were finally selected for this study. 
The figure below is a PRISMA representation of the related results obtained from the cognitive- and instructional-based search (Fig. 1).

\section{Mobile learning}

Mobile learning is a way of accessing learning materials through a mobile device irrespective of traditional learning settings. Considering its mobility as a key advantage, it allows for pervasive learning and enhances interactions between students and tutors for better learning experiences. Online and offline learning can happen anytime and anywhere. Learners can additionally interact with learning contents in real time. Mobile phones are equipped with a wide set of sensors through which important data are collected and processed. Although multiple functions which were not supported in the past are now available in mobile devices, the need to fully exploit information sensing using a smartphone will significantly improve mobile learning (Foti and Mendez 2014; Manakil and George 2017; Bernacki et al. 2020).

Using the advanced techniques present in mobile technology, observations around the surrounding can be detected which ultimately allows for adaptive learning contents to be generated. This new learning paradigm promotes an engaging learning style where learning happens in the dynamic and constantly changing environment. To facilitate this learning approach in mobile learning, the main learning theories cannot be disregarded. Learning theories normally fall into three categories, namely the Behaviourism which concerns the moods and state of the mind of the learner, the Cognitivism which responds to the different senses of the learner and finally the Constructivism which considers the prior knowledge of the learner (Parsons and MacCallum 2020). The behaviourist learning theory has been extensively investigated. However, emotional factors of the learner have been given very little attention (Liu et al. 2020) in mobile learning, especially when environmental and biological contexts (motivational impacts of the learner or their behaviours) are involved (Cavus and Ibrahim 2009; Feldon et al. 2019). To investigate emotional presence in

Fig. 1 PRISMA representation of search conducted

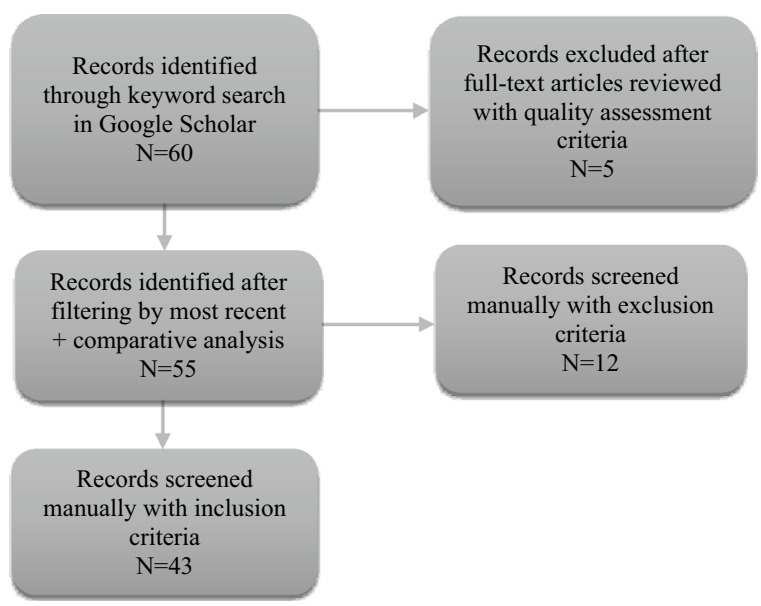


mobile learning, the aim is to identify any key design elements which can enhance the learning pattern of an individual.

Another attribute to consider for mobile learning is the instructional design principles. Poorly designed courses increase confusion, frustration and discourage learners. Viewing contents on small screen devices compared to larger screens are two distinct experiences. Physical characteristics of the device such as the screen sizes, layout of devices, platforms compatibility, bandwidth and sensors availability need to be considered as well as the user context to meet the requirements for appropriate instructional designing. Therefore, using instructional design strategies keeps in equilibrium the cognitive burden of the learner and eventually improves learning in an authentic surrounding (Merriënboer et al. 2002; Chu 2014).

With an appropriate flow of enriching learning elements and simplistic instructions, learners can succeed in deriving their meaning based on individual experiences. In this way, learning in different surroundings and among a broad range of devices will be supported.

\section{Self-regulated learning (SRL)}

Self-regulated learning (Ben-Eliyahu and Bernacki 2015) is a learning concept where the students plan, set some specific objectives and devise strategies to complete particular academic practices. With a mobile phone at hand, learners can easily connect to educational apps anytime and control or monitor their activity-based performance. Moreover, features such as learning analytics served on mobile-assisted systems based on contextual data allow tracking and enable self-learning ability per individual. In this way, learners are allowed to determine their progress over time. The Tin Can API or xAPI (Chiang et al. 2015) is an appropriate example of an activity-based process. It tracks and stores the student's performance during a learning session. These data are available both online and offline, thus allowing the students to be self-aware, selforganized, self-motivated and to better understand their academic performances.

Besides, social networking environment such as Facebook and Twitter is known to be a highly engaging tool. It allows sharing of learning contents instantly, participation in chat forums or webinars for knowledge exchange and is seen to be enhancing realtime interactions under distinct situations. As students tend to be regularly connected to social media platforms, learning is conducted in a self-paced mode with advanced interactions through online communities.

\section{Cognitive load principles for mobile learning}

Cognitive load can be defined as the total amount of strain that the mental capabilities of a learner exert while studying. Meaningful learning involves cognitive processes. Cognitive load theory (CLT) is a schema which explores the working memory capacity of a learner for proper learning (Sweller 1994). It is classified into intrinsic, germane and extraneous loads. The intrinsic load represents complex information. It is the amount of information the working memory of the learner 
deals with simultaneously when learning occurs (Hwang et al. 2013). Germane load describes the load which allows learners' conscious focus of attention to understand and recall the learning contents. It also encourages the growth of knowledge transfer (Sweller et al. 1998). Finally, the extraneous load consists of all the irrelevant, insignificant learning elements which cause added stress on the memory load of the learners. Improper use of instructional design can increase the mental processes leading to high extraneous cognitive burden (Cierniak et al. 2009). However, recent studies (Korbach et al. 2018) suggest considering the updated version of the cognitive theory which consists of only the intrinsic and extraneous loads as the main types.

Cognitive load theory recommends the extraneous load to be reduced by reengineering learning activities when the intrinsic complexity of a task remains fixed (Sweller et al. 2011). Simultaneously, when the extraneous cognitive load decreases, the cognitive resources filtered as germane should balance the load as illustrated in Fig. 2.

A good way to reconstruct the display of learning elements is to limit repetitive learning elements or confusing statements (Hwang et al. 2013). Learning contents delivered on mobile phones are often quite complex and therefore require deeper mental efforts from the learner since cognitive load theories are not yet well applied in mobile learning systems (Sepp et al. 2019). Different foreign language mobile learning applications have tried to manage the cognitive load to improve learning (Chen and Chung 2008; Chen and Lin 2016; Hsu 2011). However, cognitive load in mobile learning platforms has received very little attention and further works are required in this area. Cognitive management in mobile learning systems is important since cognitive features such as the level of concentration, learning capabilities, the attitude of the learner are common attributes that should be taken care of for proper adaptation of learning contents in mobile devices.

\section{Cognitive load theories}

Improper design of learning materials in mobile learning can be considered as one of the key reasons for the cognitive burden (Chu 2014). Several techniques have been suggested to allow learners to efficiently apply their previous knowledge to the situated contexts using limited working memory capacity. The following theories highlight the different relevant cognitive processes and their contributions to mobile learning.

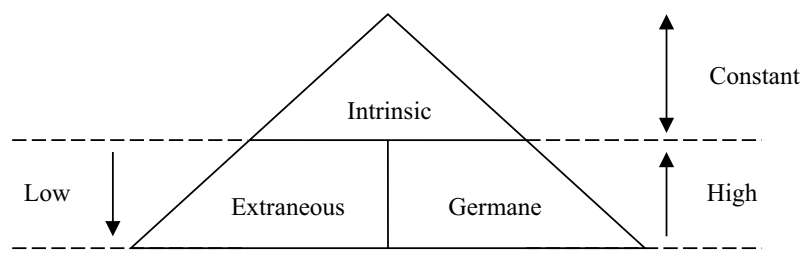

Fig. 2 Cognitive Load Theory (CLT) 
Different learners come from diverse societal backgrounds with a broad range of experiences. Their information processing techniques, perceptions, emotional states and attitudes and problem-solving methods are all distinct. PLP instructs students to use distinct styles adapted to them. Thus, PLP may be used to define the best-suited mobile learning options of learners. PLP also emphasizes on the fact that fun learning is an important criterion that should be considered. Fun learning environments and learning elements will help more in assimilation during the study process. The learner's past skills and experiences with the new learning plan elicit significant and enjoyable knowledge transfer.

\section{Cognitive information processing (CIP) theory (Mayer 2003)}

Cognitive Information Processing (CIP) theory focuses on how learners react to the surrounding conditions, processes incoming information while considering their knowledge to store new facts in memory. CIP consists of sensory memory that holds information associated with the vision and hearing senses. Meaningful learning requires the learner's prior knowledge combined with the above-stated memories to accomplish a specific task wisely. Learners can activate selective attention where they choose and process only important information while ignoring extraneous ones. Moreover, the theory states that learning elements should be organized and chunked to increase the working memory capacity in mobile learning systems. To prevent information loss during this phase, more attention to vital details as learning objects is filtered to deliver only the important ones. To this end, hierarchy diagrams, flowcharts, concept trees and many other techniques are available to support the delivered mobile learning contents. Furthermore, ending each learning session with multiple choice questions or self-assessment can be useful to recall topics learned.

\section{Levels of processing theory (Craik and LockHart 1972)}

Levels of processing theory state that the learner's mind processes stimulus information at multiple levels. The nature of the information impacts directly on the processing level. Shallow processing involves repeated activities or just reading of brief descriptions of the learning contents. It is noted that the learner's memory captures the physical traits of the information (images) and encodes auditory learning elements presented. As a result, mobile learners develop their analytical skills and derive their understanding from the concept presented which leads to long-term knowledge retention. Moreover, mobile-assisted learning if integrated with selfguided assignments after ending a specific course allows mobile learners to reflect and explore more in-depth through their understanding to increase their knowledge retention. 
Padagogy wheel

The Padagogy wheel can be regarded as a flexible tool which allows teachers to determine which application best suit their learning sessions. Sections in the wheel which were pre-defined with the four important domains of the Bloom's Taxonomy are now complemented with the SAMR model (Substitution, Augmentation, Modification, Redefinition). The Padagogy wheel consists of five areas which are the graduate attributes and capabilities, motivation, Bloom's Taxonomy, Technology enhancement, and the SAMR model. This theory is designed to help tutors to systematically plan and associate each learning classes to the best-suited application which will keep students focused. This newly defined approach is flexible enough to allow cognitive load integration, long-term learning objectives in mobile systems and monitor the motivation of the learner.

\section{Missing data algorithm in mobile learning}

With the broad array of functionalities that hold novel mobile platforms, situations may arise when some instabilities occur during a learning session. This may cause missing data. As mobile learning platforms are gradually experimenting with simple human-centric activity recognition during a study session, the need to cater for missing information is reasonable. Some algorithms' examples dealing with these issues are imputing missing values using linear regression technique (Mostafa 2019) to equate the learning mechanism with comparative datasets; the Neural Saliency algorithm guide bi-directional visual perception style transfer (Zhu et al. 2020) which tries to bring consistency in the way learners perceive imagery. Images are given special attention by learners differently, and this algorithm tries to validate an overall uniform visual perception; AntLP: Ant-based label propagation algorithm for community detection in social networks (Hosseini and Rezvanian 2020) which tries to find out the similarities in datasets to provide an optimized community to fill the gap during a learning session; TDD-Net (Ding et al. 2019) puts forward a tiny defect detection network using the clustering technique to build relationships between hierarchies of events to match a suggestion for the missing information and the Channel-wise attention model-based fire and rating level detection in video (Wu et al. 2019) which associates artificial intelligence in learning models to detect fire in the surrounding. All these algorithms are aiming to work accurately with activity-based gestures of the individual and associated context and cognitive datasets to provide an automated and blended system. The challenge is real and complex, but integrating these set of rules in mobile learning could certainly provide impressive learning performance. 


\section{Learning effects of cognitive load theory}

\section{The split attention principle}

Through the use of small screen devices, it is usually hard to capture the full attention of the learner. Moreover, reprehensible design in learning materials causes a high cognitive burden and negatively affects the mobile learning experience. One possible solution to this issue is the use of the Split Attention principle which states that people learn more when the information presented is in more than one form (Mayer and Moreno 1998). Learning materials can be textual, pictorial, animated or verbal (Huang et al. 2012). To provide better mobile learning experiences, it is vital to produce instructional elements to the learner in synchronization with frequent change in the context of the learner. Ultimately, it encourages learners to process information quickly. However, too many dissimilar learning materials if presented under one interface can cause confusion, a shift in attention and hence result in poor performance for the learner.

\section{The modality effect}

The modality effect (Sweller et al. 1998) states that it is better to recall information when it is presented with the presence of an auditory channel. For example, a lesson with visual representation together with an audio or text transcript is more effective than the same pictorial with no other supported modes. Elaborative audio or text will eventually add details to support the learning process in mobile learning systems and learners with low working memory capacity can take advantage of this effect. The usage of mixed media has the potential to create an impactful learning condition as the learning resources can be combined and made dynamically available independent of the location and time. In this way, it does not obstruct the focus of the learner and therefore promotes good integration of cognitive load theory.

\section{The redundancy effect}

The redundancy effect (Sweller 2005) aims at providing only learning materials that are necessary for a learner to remember. In cognitive load theory, it is often listed as the extraneous load because of the irrelevant and excessive learning elements which generally cause an added strain on the working memory of a learner. Reducing complexities in learning materials delivered can be considered as an optimized process in promoting comprehensible learning in a mobile environment. Moreover, as mobile devices are smaller in sizes, the idea is to be able to manage the loading time and allow flexible deliveries of the learning contents to engage a good learning flow.

\section{The worked example effect}

A step by step guide of performing particular tasks is called the worked example effect (Sweller 2006). This cognitive load theory effect is commonly used in 
complex problem-solving learning. The worked example effect handles mostly the germane load of the CLT to provide a maximum storage capacity for information so that learners can eventually construct meaningful evidence for self-learning at their own pace.

\section{The seductive details effect}

In the seductive details effect (Harp and Mayer 1998), pieces of information are transformed into remarkable and interactive learning elements to make a course content interesting. However, with the presence of extra details in the lessons, seductive details often add extraneous cognitive load and this results in poor learning performance.

The aim of cognitive management in mobile learning is to facilitate learning by providing non-complex learning elements. From the above-mentioned cognitive load theories and learning effects, criteria such as originality, the importance of learning contents, prior knowledge or experience, comprehension of the learner and presentation or instructional design are considered for evaluation. Based on the cognitive load theory's empirical evidence from Fig. 2, Table 1 defines an evaluation significance of the types of cognitive loads for the respective criteria.

\section{The efficiency of cognitive load theories for mobile learning}

According to the theories evaluated in Sects. Cognitive load principles for mobile learning and Learning effects of cognitive load theory and based on the findings of some relevant research papers (Fu and Hwang 2018; Chung et al. 2019; Yang et al. 2020; Tu and Hwang 2020), the below theoretical assumptions are made. Using a mobile device as the main asset, this shows how the principles and theories for

Table 1 Information flow for learning criteria affected by the types of cognitive loads

\begin{tabular}{|c|c|c|c|c|}
\hline \multirow[t]{2}{*}{ Criteria } & \multirow[t]{2}{*}{ Description } & \multicolumn{3}{|c|}{ Types of cognitive load } \\
\hline & & Intrinsic & Extraneous & Germane \\
\hline \multirow[t]{2}{*}{ Originality } & New information, ideas, solutions & Average & Average & Average \\
\hline & Repetition of what is said & Average & High & Average \\
\hline \multirow[t]{2}{*}{ Importance } & Important points & Low & Low & High \\
\hline & Unimportant issues & High & High & Low \\
\hline \multirow[t]{2}{*}{ Previous knowledge/experience } & $\begin{array}{l}\text { Relevant statements based on } \\
\text { previous knowledge }\end{array}$ & Low & Low & High \\
\hline & Irrelevant statements, diversions & High & High & Low \\
\hline \multirow[t]{2}{*}{ Comprehension } & Clear, unambiguous information & Low & Low & High \\
\hline & Confused statements & High & High & Low \\
\hline \multirow[t]{2}{*}{ Presentation/Instructional design } & Clear content presentation & Low & Low & High \\
\hline & Unclear presentation & High & High & Low \\
\hline
\end{tabular}


cognitive load management require collaboration from both the learning contents and the learner's personal traits to devise appropriate adaptation strategies. The learning contents are categorized under the learning material, the learner's personal traits form part of the learner's capabilities and the value for adaptation strategies is regrouped under the learning effectiveness. The flow is as follows: (1) the mobile device is the integral tool, (2) the learning contents adopt good use of instructional design and principles together with the learning theories, (3) and finally the learner's personal traits can experience satisfaction while learning occurs.

Based on the theoretical assumption from Fig. 3, a learning efficiency model chart is derived. Three main categories are considered, namely the learning material, the learning effectiveness and the learner's capabilities. Each of the factors is described in Table 2. A comparative analysis of the different cognitive load theories listed from Sect. Cognitive load theories is then presented in Table 3 based on the learning efficiency model chart.

\section{Learning efficiency model chart}

Based on the theories and learning effects for cognitive load described in Sects. Cognitive load principles for mobile learning and Learning effects of cognitive load theory, respectively, some key abstractions are derived based on three basic categories: learning material available, learning effectiveness and the learner's capabilities. These are based on both the internal and external values of the object model. Internal values relate to the learner's traits, for example, previous knowledge, moods or interpersonal skills and external values qualify the learning elements such as the

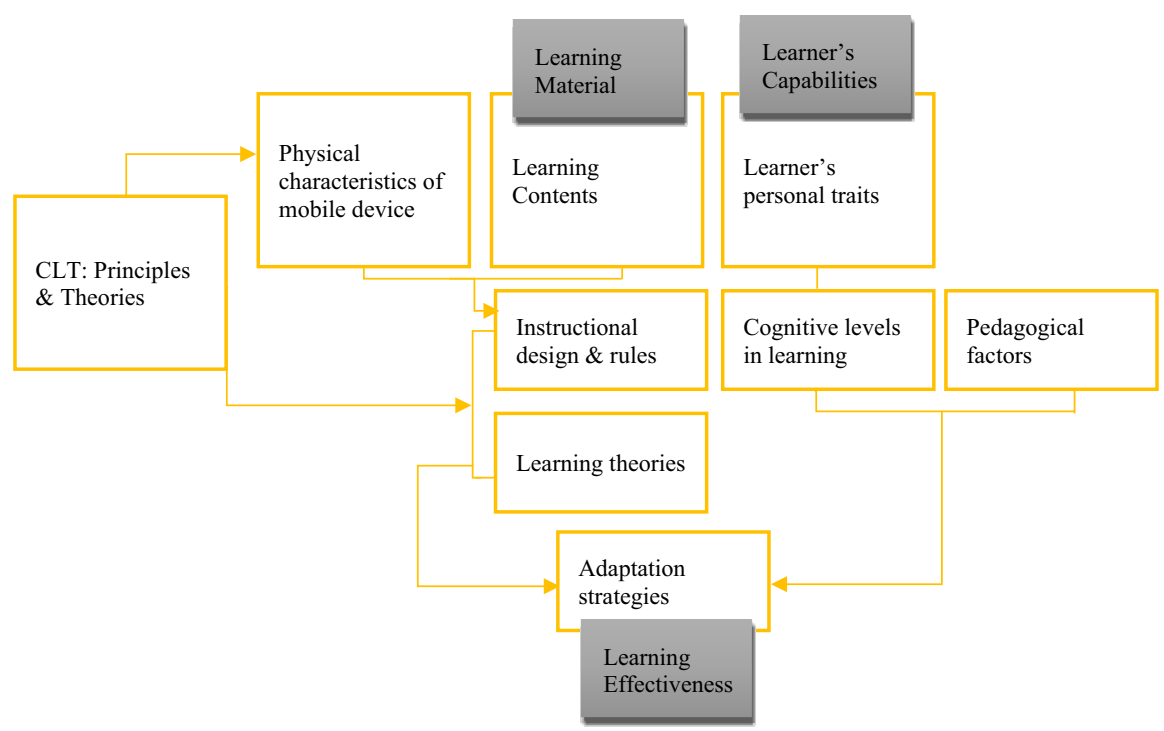

Fig. 3 CLT theoretical assumption for efficient learning 


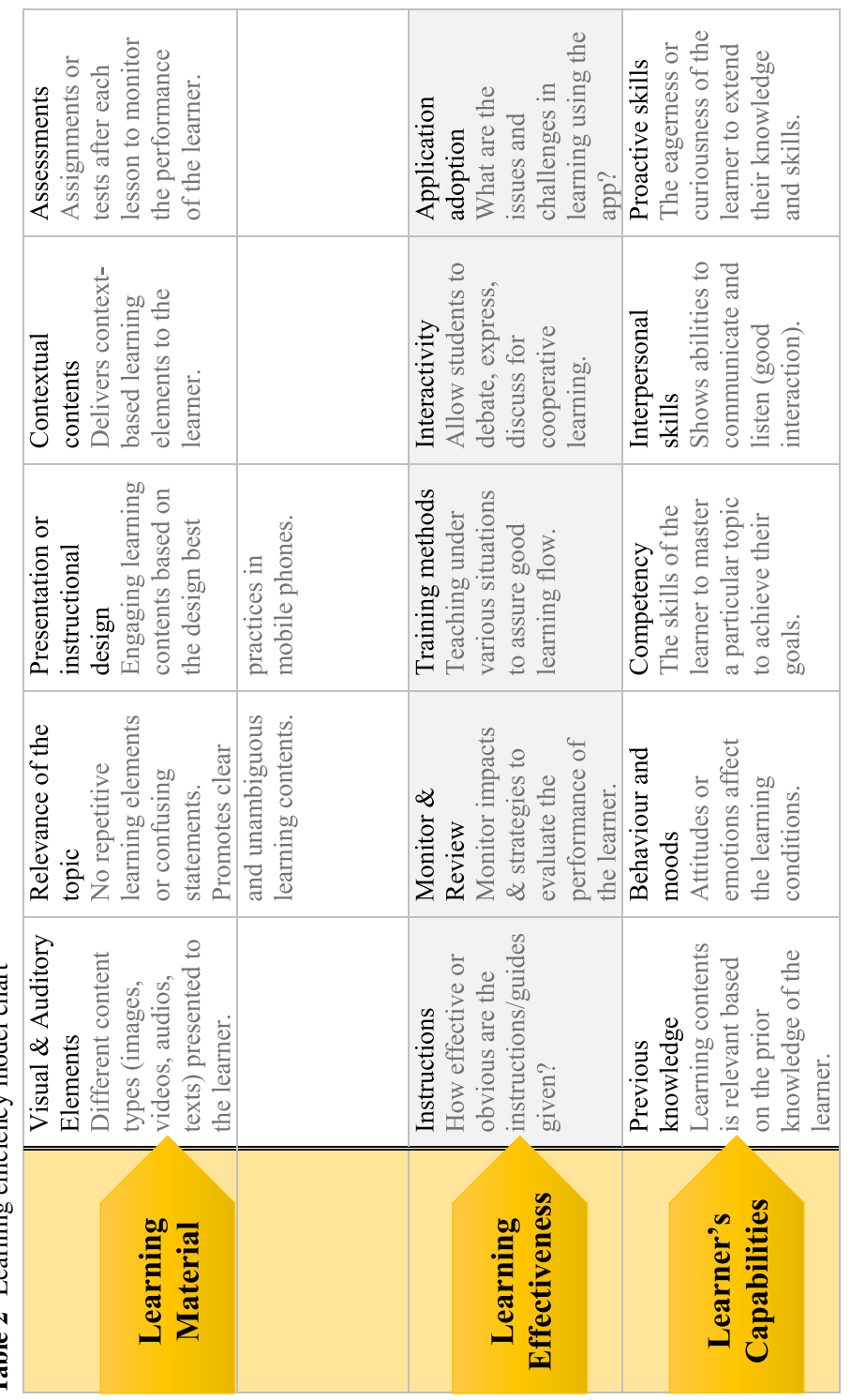




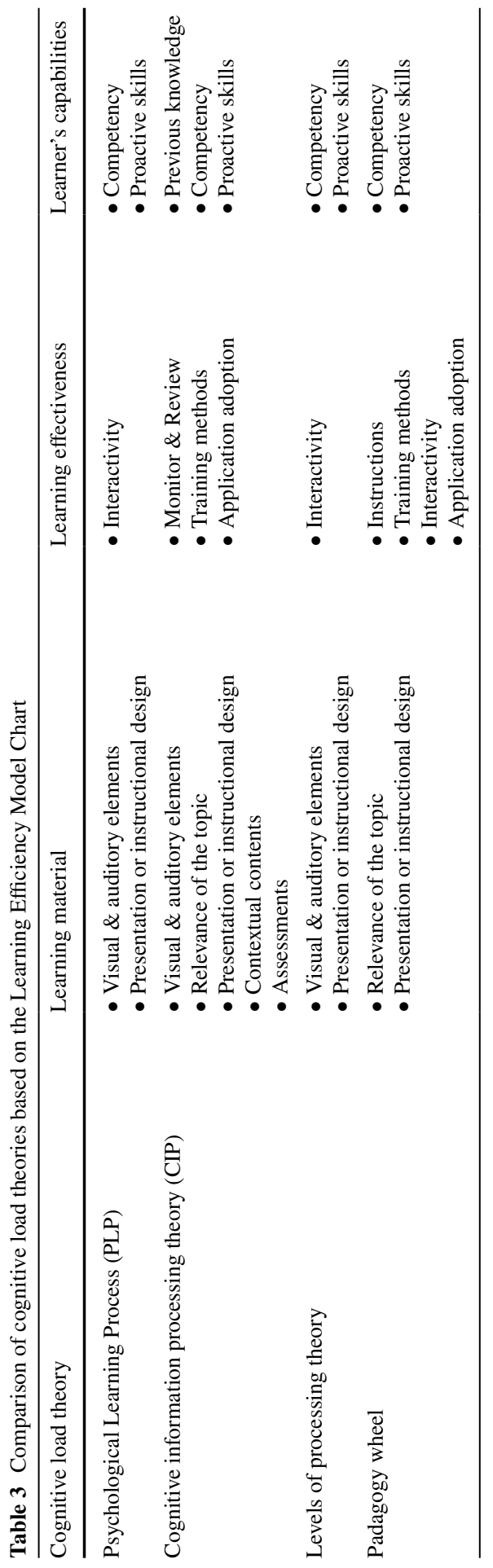


visual or auditory components or assessment methods, etc. The dataset is presented with short descriptions in Table 2.

\section{Comparison of cognitive load theories based on the learning efficiency model chart}

The cognitive load theories from Sect. Cognitive load theories are compared using the different combinations of the learning efficiency model chart from Table 2 . This comparative assessment is detailed in Table 2.

It is observed that most cognitive load theories make use of different multimedia assets (texts, images, sound and animation) to deliver information-rich contents. These contents are delivered using different multimedia channels. Also, effectiveness in learning is refined with past experiences and appropriate selection of learning contents based on the learners' preferences. In this way, learners can define their understanding, take advantage of being more autonomous and organized in choosing their self-adapted and preferred learning formats. Moreover, using a casual and animated, the fun-loving interface makes learning enjoyable and learners feel more inspired to study.

However, concern about the integration of factors governing the learning effectiveness and the learners' capabilities to reformulate learning elements in mobile learning is limited. To allow adaptive learning to occur and to thrive in this mobiletech world, the internal contexts about the learner such as the emotional traits, the behaviour and moods, interpersonal or proactive skills, the assimilation and reaction, perception, interaction, application adoption and so on are important factors to consider. These factors should be made more accessible to reform and personalize instantly the learning conditions of the learner and at the same time promote self-regulated learning based on contextual situations. Self-efficacy in learning can further be split using the micro-learning concept which tries to deliver learning materials in small chunks. It eliminates repetitive learning materials or any confusing statements and promotes clear learning patterns. Besides, this learning method addresses the cognitive deficiencies in learners and allows long-term memory recall through distinct behaviours and attitudes. As a result, there will be more positive responses as learners selectively choose and process only important information.

\section{Instructional design principles for managing cognitive load in mobile learning}

Many instructional design strategies are proposed to keep in equilibrium the cognitive burden and to eventually improve learning in an authentic surrounding (Merriënboer et al. 2002; Chu 2014). Instructional design for mobile learning helps in constructing a space for learning by structuring content and creating activities that engage mobile learners and ease learning. Its importance in remote learning is noteworthy since a student's learning experience is mediated through different technological forms. Instructional design strategies were proposed to keep in equilibrium 
the cognitive burden and to eventually improve learning in an authentic surrounding (Merriënboer et al. 2002; Chu 2014).

It is important to provide suitable contents with a good presentation that fit the environment of distinct mobile interfaces. Designing learning contents for mobile devices requires a well-structured plan and good knowledge about the devices. Poorly designed courses increase confusion, frustration and discourage learners. Moreover, viewing contents on small screen devices compared to larger screens are two distinct experiences. Physical characteristics of the device such as the screen sizes, layout of devices, platforms compatibility, bandwidth and sensors availability need to be considered to meet the requirements for appropriate instructional designing (Liu et al. 2014). Besides, connectivity issues and situated context are important considerations of mobile learning (Sharples et al. 2005; Kukulska-Hulme et al. 2009).

Moreover, multimedia support is key to transform learning into content-rich interactive sessions (Huang et al. 2012). The Cognitive theory of learning in Sect. Cognitive load principles for mobile learning evokes that mobile learners study well when information is presented in more than one media (texts, audio or visual). Thus, while designing learning activities, instructional designers need to consider the environment in which learning is going to take place (Shadiev et al. 2015; Briz-Ponce et al. 2017). Mayer and Moreno (2003) describe an "integrated presentation" concept which allows learners to be more focused on essential information flow and thus optimize their cognitive capacity. In this section, some of the most used instructional design strategies to support and achieve meaningful learning independent of the learner's actual situation are discussed.

\section{Instructional design techniques}

The following section describes some of the instructional design models and techniques widely adopted for the development of mobile learning contents. The models are analysed using the cognitive load principles to see how learning can be optimized at different levels.

\section{The ADDIE model}

The ADDIE (Wang and Shen 2012) model addresses the designing issues in learning systems under five stages: analysis, design, development, implementation and evaluation. It represents a dynamic and flexible guideline which helps instructional designers to perform systematic construction of what is needed to output comprehensive and targetable learning materials. It regroups clarifications, instructional goals and objectives that need to be established for appropriate learning. Characteristics such as the learner's actual environment, prior knowledge and skills are identified to evaluate the pedagogical levels. This model helps in identifying the knowledge gap and analyses the creation of a mobile learning system to set appropriate learning objectives with regard to cognitive load theories. Moreover, it considers adding a training plan which eventually acts as a guide to learners, giving additional 
instructions for proper self-managing learning conditions. With its responsive ability to work well under various screen sizes for mobile phones, this model is seamless in hybrid learning and is seen to be very popular among online courses, hence an advantage to mobile learning.

\section{Universal design for mobile learning (UDL)}

Universal Design for Learning (UDL) (Rose 2000) is a framework which assists in the design of instructional learning elements and caters for individuals' learning dissimilarities. It includes four components which are goals, methods, materials and assessments. In mobile learning, the UDL guidelines look over three specific principles, namely representation, expression and engagement. Representation offers mobile learners multiple ways to acquire information and understanding. Expression provides learners with choices to explore and demonstrate their previous skills and knowledge and engagement elicits the learners' interests in learning.

UDL recognizes that learners possess different skills, experiences and learning styles, and tries to engage them with a desirable learning process. It emphasizes flexible and customizable contents using multiple modes of delivery. Moreover, the elementary customizable mobile learning interfaces help them to stay focused on the learning contents. They can eventually evaluate their performances through assessments. Elias (2011) elaborates on the Universal Design for Mobile Learning Principles as follows:

Equitable The design is simple, smooth and reachable for mobile-assisted learners with diverse abilities and backgrounds, independent of their current locations.

Flexible Learning design contains a wide range of abilities and preferences. Learners can choose their best-suited methods.

Simple and intuitive use The simple content interface promotes trouble-free learning regardless of the user's prior knowledge and the observable changes in moods.

Perceptible information Rich information is communicated effectively to the learner, even for users with sensory impairments (vision problems and reading disabilities).

Tolerance for error Minimizing the adverse consequences of mistakes in the design.

Minimum physical and technical effort The design should be effortlessly used with reduced physical and mental fatigue.

Support of a community of mobile learners Learning-interaction among peers and the connected mobile learners are beneficial for group learning.

Feedback or comments The instructor's comments or feedback on specific assignments are comprehensive and straightforward.

The UDL comes with assistive architecture to aid in delivering learning contents with appropriate cognitive load principles for distinct learners in mobile devices. A concrete example of this feature is delivered in a mobile app loading contents related to arithmetic (Tobin and Behling 2018). Learning objects are designed in an adaptively simplistic format to match criteria that support the cognitive load theories as well as instructional design strategies. 


\section{The ARCS model of motivational design theories}

The ARCS (Attention, Relevance, Confidence and Satisfaction) motivational design process (Keller 2010) is a methodical problem-solving approach that requires the learners' knowledge and motivation. In the learning process, the mobile learner would want to benefit from the most effective knowledge transfer for the chosen topic. An effective instructor should be able to gain a learner's attention and keep it throughout the course. For this reason, instruction should be designed to improve the following four learner motivation categories:

Attention (A) Alert the learner's interest and awareness.

Relevance $(R)$ Relate to the learner's previous experiences, skills and needs.

Confidence $(C)$ Support the learner's achievement for relevant objectives.

Satisfaction $(S)$ Understand the learner's sense of reward for success.

The development includes the identification of appropriate elements to support the learners' motivation. The analysis of the learners' characteristics is mandatory to assess their motivational prerequisites which are used to identify influencing approaches to encourage effective learning. Self-study relies on the effectiveness of the instructional-designed learning materials to match the learner's study style and comprehension and hence contributes to successful learning outcomes.

The challenge in mobile learning is to optimize the use of portable devices to make learning practices more productive. The following general guidelines for the implementation of mobile learning contents and resources are as follows:

The Platforms and Formats Design and development of mobile-based applications should cater for cross-platform accessibility and compatibility. Resources which can be opted for both mobile and non-mobile platforms should be taken as a primary choice which will eventually boost up the development of the learning applications to another level. Developers can, therefore, optimize defined modules so as they are adapted to both desktop PC and mobile interfaces.

Delivery, Accessibility \& Usability Applying user-centric definitions instead of a content-centric mindset will help developers to apply appropriate standards for accessible, usable and exchangeable mobile-compatible contents.

Design Page layouts should be designed to prevent scrolling in two dimensions (horizontal and vertical) which are not UX/UI compliant. Learners should not experience any technical difficulty while learning using mobile devices.

The ARCS model requires the learner's performance, motivation and confidence for effective learning to take place. This highlights the fact that it works towards eliminating split attention deficiencies and therefore helps the learner to own a selfdefined learning guide. This enhances the attention of the learner towards specific learning topics and helps in optimizing the learning flow.

\section{Mobile human-computer interaction (MHCI)}

Mobile Human-Computer Interaction (MHCI) in mobile learning is concerned with the learners' actions and interactions with the mobile system under multiple contexts. Producing only a functional mobile application with properly smart-fitted contents on the small screen devices is not adequate. The physiological conditions of 
the learner which changes drastically with varied situations should be considered when building up an appropriate mobile learning application. Also, the motivation and engagement of mobile learner is another important characteristic to integrate into the core development of mobile learning applications. One suggested option is to have a personalized system which will create an engaging interaction rather than contributing to a one-size-fits-all approach. Multiple ways exist to evaluate interfaces and identify areas of improvement in existing design principles. Nielsen Norman's (1994) Usability Heuristics remains the most popular and widely used technique. The MHCI shows that learners cannot take full advantage of the learning contents they are presented with. The system neglects the cognitive load management. For an uninterrupted learning flow, additional work must be done to cater for appropriate cognitive load principles and instructional designing.

\section{Merrill's first principles of instruction}

Merrill (2002) identified five Instructional Design principles intending to promote effective learning environments. The five distinct instructional design principles that are advocated are as follows:

Task/Problem Centered Learning is more versatile when learners engage themselves in solving difficulties.

Activation Easy learning is attributed when prior knowledge is used to build new skill definitions. For example, consistency in learning objects helps the learner to recall or recognize specific statements which advocate knowledge management.

Demonstration Providing practical sessions and coaching ease the study process and promote convenient learning experiences.

Application After completing a particular lesson, learners apply the knowledge acquired which helps them to control and explain the learning facts.

Integration Integrating similar topics based on the learners' immediate surroundings accounts for a better understanding of key features.

\section{C/ID model}

The 4C/ID model (Merriënboer et al. 2002) can be described as an instructional design method that addresses concerns related to complex problem-solving. To establish efficient teaching practices, the four stages of the 4C/ID model can be useful:

The learning tasks Using mobile learning apps, constant high-end support in learning is useful for quality engagement. However, the support decreases gradually as learners acquire required knowledge and thus can independently continue the study on their own.

Supportive information It supports cognitive-related features. A learner with prior knowledge or skills about a particular learning topic will find distinct learning support for engaging interaction.

Just-In-Time information Guidelines to ease the learning task are always available when needed. Mobile learning apps can consider making use of push notifications 
instances prompting the student to resume a particular quiz after a short lapse of time.

Part-task practice It provides recurrent tasks to allow learners to reach a certain height of automaticity. For example, a moderate performance for a specific lesson assessment can always allow another attempt until the student reaches a reasonable level of knowledge acquisition.

\section{Discussions}

Adapting learning contents through heterogeneous devices is still a challenge. As seen from the above analysis, different instructional design techniques have focused on different adaptive conditions for better accessibility and usability. Dynamic control is present in the learning contents but little focus applied to the user context. No emotional traits are observed for cognitive assessment. The behaviour and active reactions of the learner form important concerns. For instance, decisions of the learner could be dependent on his mood. Learners are often presented with learning materials that may not be appropriate to their learning style when no cognitive rules are defined. This causes some extra efforts in the working memory of the learner limiting to mental stress.

The instructional design models provide a common learning interface to learners and it is difficult to customize learning activities as per preferred preferences. Therefore, applying learning effects for cognitive loads give learners the ability to create their own learning spaces based on prior experiences and preferences and will allow the development of a personalized and self-paced learning environment. Moreover, relevant context-aware parameters to manage cognitive issues in mobile learning need a deeper investigation. Making decisions based on actual circumstances and adapting learning in real time have an impact on the learner. The aim is to make learning flow easier and engaging on mobile devices under distinct situations. Indeed, the development of adaptation strategies that adapt to mobile learning contents based on the cognitive level of the learners is still an unexplored research area.

One interesting thought of bringing forward fun learning is being able to manage learning in a social networking platform. The time spent with peers or connected people while engaging serious and meaningful interactions or participating in group events can be efficient. Promoting this spontaneous and flexible appreciation in the learning conditions is another target in mobile learning. Therefore, good association and integration of cognitive load principles with instructional design strategies and learning effects will ultimately reduce the ambiguity in the representations of learning resources, and enhance understanding and critical thinking. A pedagogical component needs to be developed that assesses the psychological and behavioural aspects of individual learners and allows for the provision of distinct learning styles adapted to the learners. Delivering consistent and organized learning contents based on pedagogical elements will allow a comfortable learning pattern in mobile learning.

In Table 4, the available components from the cognitive load theories described in Sect. Cognitive load principles for mobile learning are associated with the learning 


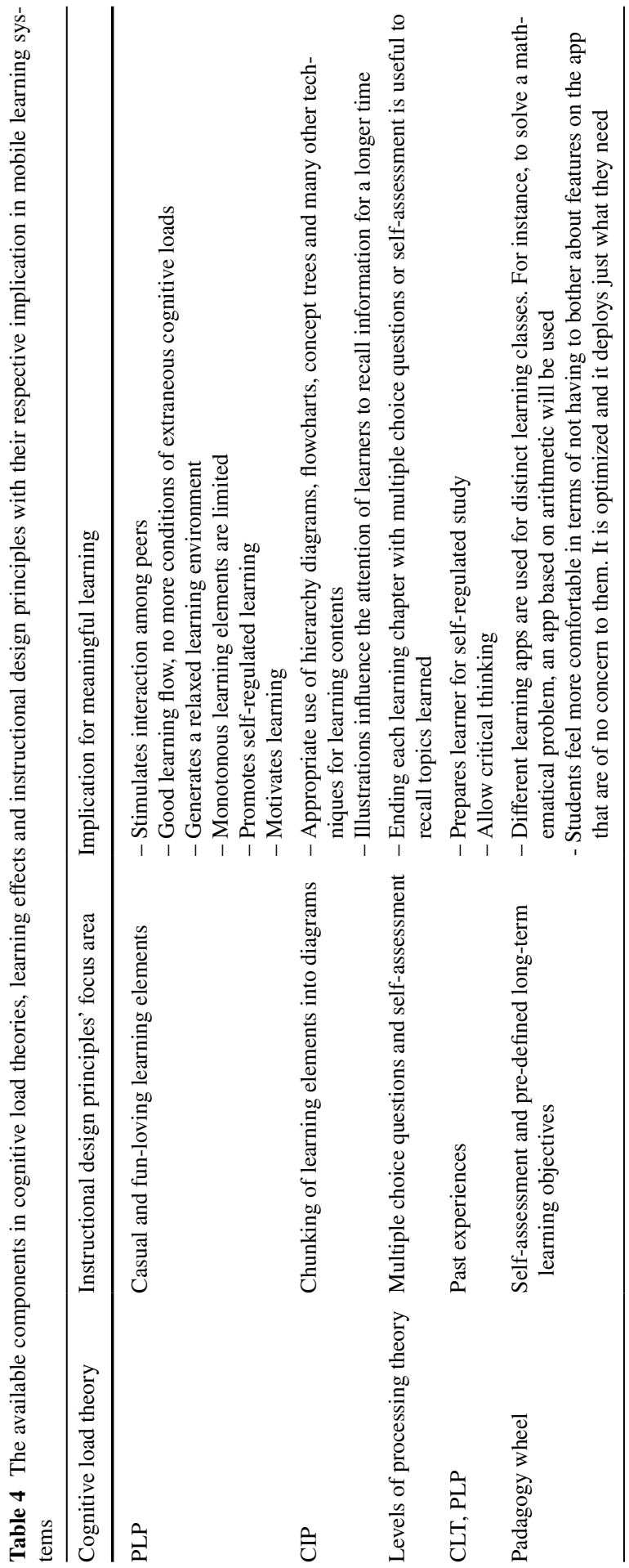


effects and the instructional design principles focus area to evaluate their respective implication for meaningful learning in mobile platforms.

As seen from Table 4, for each of the cognitive load theory, different learning effects and instructional rules were applied to readjust the learning contents and conditions. The focus areas are all different for the cognitive load theories listed. The need to recognize one cognitive load theory which will combine all the distinct parameters will be a challenge in adapting the learning elements based on the instructional rules.

The following statements in Table 5 outline how the available components and learning implications from Table 4 for each of the cognitive load theory can reduce the types of cognitive load as described in Sect. Cognitive load principles for mobile learning.

As seen in Table 5, most of the cognitive load theories match the evaluation parameters set for the types of cognitive load in Sect. Cognitive load principles for mobile learning. Most of the theories provide relevant information and good layout presentation. The low or average ratings attributed to the types of cognitive load defined in this table indicates that there is good management of the use of cognition, learning effects and the instructional principles across each theory. However, with its dynamic motions, further investigation to mitigate better responses from the user needs to be done (Table 6).

From the above statements, it is clear that cognitive theories should be incorporated in mobile learning systems which will ultimately intend to reduce the ambiguity in the representations of learning resources, and enhance understanding and critical thinking. Moreover, a pedagogical component needs to be developed that assesses the psychological and behavioural aspects of the individual learner and allows the provision of distinct learning styles adapted to the learner. More dynamic adaptation is required which considers the user-centred context. Also, the possibilities to merge both the physical and user contexts with cognitive and instructional disciplines to deliver adaptive and personalized learning materials should be investigated. Students should be provided with choices to adapt the learning contents based on their prior experiences and behaviours to develop their learning patterns.

\section{Conclusion}

Building an effective mobile learning system which can be accessed anytime and anywhere to help students to learn based on their learning style and pedagogical reflections, actual behaviour and surrounding conditions is challenging. It is observed that currently, mobile learning systems are not able to harness the broad array of powerful abilities that modern mobile devices possess to deliver appropriately adapted mobile learning elements. The exploration of cognitive load management together with the learning effects and the instructional design principles for the provision of adaptive and personalized learning using situational, physical and pedagogical contexts is currently limited. More flexible opportunities could be given to the learner while adapting mobile content delivery to existing instructional design theories, including distinct multimedia assets for effective learning performances. 


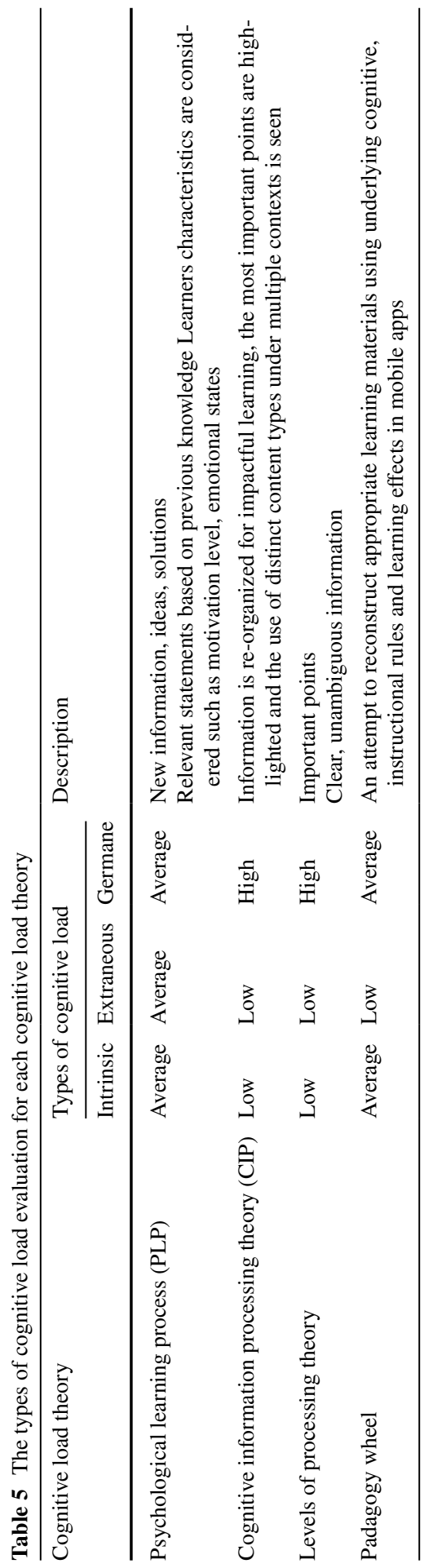


Table 6 The limitations of existing cognitive load theory \& instructional design principles with the potential benefits for mobile learning systems

Limitations of cognitive load theory \& instructional design principles for mobile learning systems

Limitations $\quad$ Potential benefits if limitations are resolved

Pedagogical setup

Assess the psychological and behavioural aspects of the individual learner

Allow for the provision of distinct learning styles adapted to individual learners

Behavioural aspects of the learner Giving more attention to behavioural learning will allow constant observation of the learner and deliver learning elements based on the current mood

Context-awareness

Combining cognitive load and instructional design with contextawareness in mobile learning will improve learning based irrespective of the conditions

No emotional traits

Individual differences in behaviour to enable better interaction during the study

Identify the intention of the learner to deliver personalized learning contents

Personalization

Incorporation of multiple options of learning contents to meet the needs of individual learners

Adaptive learning activities

Engage and motivate learners to achieve their goals as per their terms and conditions

Moreover, none of the theories addressed in this paper discusses the introduction of animations during study though it is a growing trend which looks favourable in mobile learning. Also, based on the wide adoption of technological infrastructure such as the cloud-based, multimedia, portals, API gateways, implementing animated learning elements to mobile learning can be considered as an innovative upgrade. To this end, cognitive load strategies and techniques need to focus on mobile learning framework that exploits the various animation trends.

As future works, the learner's internal context data should be extensively exploited combined with the physical characteristics of mobile devices to allow instant and logical interpretations of information. The option to personalize the learning environment based on the learner's ability and introducing deep artificial intelligence techniques to monitor both the psychology and performance of the learner are promising. Moreover, with the outbreak of COVID-19, many countries have been put into lockdown to stop the spread of the virus. This urges the need for educational systems to find alternatives and promote remote learning strategies worldwide. Different approaches in deep learning have been identified using training models and neural networks for specific contextual datasets (Jaiswal et al. 2020; Shukla et al. 2020), image encrypted techniques based on chaotic maps (Kaur et al. 2020; Gupta et al. 2020) and image fusion theories (Kaur and Singh 2019). Adjusting learning contents collectively with context-aware reactions, cognitive modules and design principles with these key methods could define better algorithm across the mobile learning platforms and help learners to concentrate more on their abilities to reach out a constructive learning performance. This approach will ultimately 
reduce the ambiguity in the representations of learning resources, and enhance understanding, critical thinking, interactions and instructional definitions.

\section{Compliance with ethical standards}

Conflict of interest The authors declare that they have no conflict of interest.

\section{References}

Baddeley, A. D., \& Hitch, G. (1974). Working memory. Psychology of Learning and Motivation, 8, 47-89.

Beavers, A. S., Lounsbury, J. W., Richards, J. K., \& Huck, S. W. (2013). Practical considerations for using exploratory factor analysis in educational research. Practical Assessment, Research, and Evaluation, 18(1), 6.

Ben-Eliyahu, A., \& Bernacki, M. L. (2015). Addressing complexities in self-regulated learning: A focus on contextual factors, contingencies, and dynamic relations. Metacognition and Learning, 10(1), $1-13$.

Bernacki, M. L., Greene, J. A., \& Crompton, H. (2020). Mobile technology, learning, and achievement: Advances in understanding and measuring the role of mobile technology in education. Contemporary Educational Psychology, 60, 101827.

Briz-Ponce, L., Pereira, A., Carvalho, L., Juanes-Mendez, J. A., \& Garcia-Penalvo, F. J. (2017). Learning with mobile technologies-Students' behaviour. Computers in Human Behaviour, 72, 612-620.

Cavus, N., \& Ibrahim, D. (2009). M-Learning: An experiment in using SMS to support learning new english language words. British Journal of Educational Technology, 40, 78-91.

Chen, C. M., \& Chung, C. J. (2008). Personalized mobile english vocabulary learning system based on item response theory and learning memory cycle. Computers \& Education, 51(2), 624-645.

Chen, C.-M., \& Lin, Y.-J. (2016). Effects of different text display types on reading comprehension, sustained attention and cognitive load in mobile reading contexts. Interactive Learning Environments, 24(3), 553-571.

Chiang, C.F., Tseng, H.C., Chiang, C.C. \& Hung, J.L. (2015). A case study on learning analytics using Experience API. In Society for information technology \& teacher education international conference, pp. 2273-2278. Association for the Advancement of Computing in Education (AACE).

Chu, H.-C. (2014). Potential negative effects of mobile learning on students' learning achievement and cognitive load-A format assessment perspective. Educational Technology \& Society, 17(1), $332-344$.

Chung, C. J., Hwang, G. J., \& Lai, C. L. (2019). A review of experimental mobile learning research in 2010-2016 based on the activity theory framework. Computers \& education, 129, 1-13.

Cierniak, G., Scheiter, K., \& Gerjets, P. (2009). Explaining the split-attention effect: Is the reduction of extraneous cognitive load accompanied by an increase in germane cognitive load? Computers in Human Behaviour, 25, 315-324.

Craik, F. I., \& Lockhart, R. S. (1972). Levels of processing: A framework for memory research. Journal of Verbal Learning and Verbal Behavior, 11(6), 671-684.

Ding, R., Dai, L., Li, G., \& Liu, H. (2019). TDD-net: A tiny defect detection network for printed circuit boards. CAAI Transactions on Intelligence Technology, 4(2), 110-116.

Efklides, A. (2008). Metacognition. Defining its facets and levels of functioning in relation to self-regulation and coregulation. European Psychologist, 13, 277-287.

Elias, T. (2011). Universal instructional design principles for mobile learning. The International Review of Research in Open and Distributed Learning, 12(2), 143-156.

Feldon, D. F., Gregory, C., Juth, S., \& Jeong, S. (2019). Cognitive load as motivational cost. Educational Psychology Review, 31, 1-19.

Foti, M. K., \& Mendez, J. (2014). Mobile learning: How students use mobile devices to support learning. Journal of Literacy and Technology, 15(3), 58-78. 
Fu, Q. K., \& Hwang, G. J. (2018). Trends in mobile technology-supported collaborative learning: A systematic review of journal publications from 2007 to 2016. Computers \& Education, 119, 129-143.

Gupta, A., Singh, D., \& Kaur, M. (2020). An efficient image encryption using non-dominated sorting genetic algorithm-III based 4-D chaotic maps. Journal of Ambient Intelligence and Humanized Computing, 11(3), 1309-1324.

Harp, S. F., \& Mayer, R. E. (1998). How seductive details do their damage: A theory of cognitive interest in science learning. Journal of Educational Psychology, 90(3), 414-434.

Hosseini, R., \& Rezvanian, A. (2020). AntLP: ant-based label propagation algorithm for community detection in social networks. CAAI Transactions on Intelligence Technology, 5(1), 34-41.

Hsu, I. C. (2011). An architecture of mobile web 2.0 context-aware applications in ubiquitous web. $J S W, 6(4), 705-715$.

Huang, H.-C., Wang, T.-Y., \& Hsieh, F.-M. (2012). Procedia - Social and Behavioural Sciences, 64, 332-341.

Hwang, G. J., Wu, P. H., Zhuang, Y. Y., \& Huang, Y. M. (2013). Effects of the inquiry-based mobile learning model on the cognitive load and learning achievement of students. Interactive Learning Environments, 21(4), 338-354.

Jaiswal, A., Gianchandani, N., Singh, D., Kumar, V., \& Kaur, M. (2020). Classification of the COVID19 infected patients using DenseNet201 based deep transfer learning. Journal of Biomolecular Structure and Dynamics. https://doi.org/10.1080/07391102.2020.1788642.

Kaur, M., \& Singh, D. (2019). Fusion of medical images using deep belief networks. Cluster Computing, 23, 1-15.

Kaur, M., Singh, D., Sun, K., \& Rawat, U. (2020). Color image encryption using non-dominated sorting genetic algorithm with local chaotic search based 5D chaotic map. Future Generation Computer Systems, 107, 333-350.

Keller, J. M. (2010). Motivational design for learning and performance: The ARCS model approach. New York: Springer.

Kitchenham, B., Brereton, O. P., Budgen, D., Turner, M., Bailey, J., \& Linkman, S. (2009). Systematic literature reviews in software engineering-A systematic literature review. Information and Software Technology, 51(1), 7-15.

Korbach, A., Br"unken, R., \& Park, B. (2018). Differentiating different types of cognitive load: A comparison of different measures. Educational Psychology Review, 30, 503-529.

Kukulska-Hulme, A., Sharples, M., Milrad, M., Arnedillo-Sanchez, I., \& Vavoula, G. (2009). Innovation in mobile learning: A European perspective. International Journal of Mobile and Blended Learning (IJMBL), 1(1), 13-35.

Liu, T. C., Lin, Y. C., \& Paas, F. (2014). Effects of prior knowledge on learning from different compositions of representations in a mobile learning environment. Computers \& Education, 72, 328-338.

Liu, X., Ji, L., \& Peng, H. (2020). The impacts of task relevance and cognitive load on adults' decision information search. Aging, Neuropsychology, and Cognition. https://doi.org/10.1080/13825 585.2020.1712320.

Manakil, J., \& George, R. (2017). Mobile learning practices and preferences a way forward in enhancing dental education learning experience. European Journal of General Dentistry, 6(1), 22.

Mayer, R. E., \& Moreno, R. (1998). A split-attention effect in multimedia learning: Evidence for dual processing systems in working memory. Journal of Educational Psychology, 90(2), 312-320.

Mayer, R. A. (2003). Elements of a science of e-learning. Journal of Educational Computing Research, 29(3), 297-313.

Van Merriënboer, J. J., Clark, R. E., \& De Croock, M. B. (2002). Blueprints for complex learning: The 4C/ID-model. Educational technology research and development, 50(2), 39-61.

Merrill, M. D. (2002). First principles of instruction. Educational Technology, Research and Development, 50(3), 43-59.

Moher, D., Shamseer, L., Clarke, M., Ghersi, D., Liberati, A., Petticrew, M., et al. (2015). Preferred reporting items for systematic review and meta-analysis protocols (PRISMA-P) 2015 statement. Systematic Reviews, 4(1), 1.

Mostafa, S. M. (2019). Imputing missing values using cumulative linear regression. CAAI Transactions on Intelligence Technology, 4(3), 182-200.

Nielsen, J. (1994). Heuristic evaluation. In J. Nielsen \& R. Mack (Eds.), Usability inspection methods (pp. 25-62). New York: Wiley. 
Olmos, E., Cavalcanti, J. F., Soler, J. L., Contero, M., \& Alcaniz, M. (2018). Mobile virtual reality: A promising technology to change the way we learn and teach. In S. Yu, et al. (Eds.), Mobile and ubiquitous learning (pp. 95-106). Singapore: Springer.

Parsons, D., \& MacCallum, K. (2020). A learning theory rubric for evaluating mobile learning activities. In Mobile devices in education: Breakthroughs in research and practice (pp. 983-998). IGI Global.

Rose, D. (2000). Universal design for learning. Journal of Special Education Technology, 15(3), 45-49.

Sepp, S., Howard, S. J., \& Tindall-Ford, S. (2019). Cognitive load theory and human movement: Towards an integrated model of working memory. Educational Psychological Review. https://doi. org/10.1007/s10648-019-09461-9.

Salonen, P., Vauras, M., \& Efklides, A. (2005). Social interaction-What can it tell us about metacognitive and coregulation in learning? European Psychologist, 10, 199-208.

Shadiev, R., Hwang, W. Y., Yueh-Min, H., \& Liu, T. Y. (2015). The impact of supported and annotated mobile learning on achievement and cognitive load. Journal of Educational Technology \& Society, 18(4), 53.

Sharples, M., Taylor, J., \& Vavoula, G. (2005). Towards a theory of mobile learning. Proceedings of mLearn, 1(1), 1-9.

Shukla, P. K., Shukla, P. K., Sharma, P., Rawat, P., Samar, J., Moriwal, R., et al. (2020). Efficient prediction of drug-drug interaction using deep learning models. IET Systems Biology. https://doi. org/10.1049/iet-syb.2019.0116.

Sweller, J. (1994). Cognitive load theory, learning difficulty, and instructional design. Learning and Instruction, University of NSW, Australia, 4, 295-312.

Sweller, J., van Merrienboer, J. J. G., \& Paas, F. (1998). Cognitive architecture and 34 instructional design. Educational Psychology Review, 10(3), 251-296.

Sweller, J. (2005). The redundancy principle in multimedia learning. In R. E. Mayer (Ed.), The Cambridge handbook of multimedia learning (pp. 159-167). Cambridge: Cambridge University Press.

Sweller, J. (2006). The worked example effect and human cognition. Learning and Instruction, 16(2), 165-169. https://doi.org/10.1016/j.learninstruc.2006.02.005.

Sweller, J., Ayres, P., \& Kalyuga, S. (2011). Cognitive load theory. New York: Springer.

Tobin, T. J., \& Behling, K. T. (2018). Reach everyone, teach everyone: Universal design for learning in higher education. Morgantown, WV: West Virginia University Press.

Tu, Y. F., \& Hwang, G. J. (2020). Trends and research issues of mobile learning studies in hospitality, leisure, sport and tourism education: A review of academic publications from 2002 to 2017. Interactive Learning Environments, 28(4), 385-403.

Wang, M., \& Shen, R. (2012). Message design for mobile learning: Learning theories, human cognition and design principles. British Journal of Educational Technology. https://doi.org/10.111 1/j.1467-8535.2011.01214.x.

Wu, Y., He, Y., Shivakumara, P., Li, Z., Guo, H., \& Lu, T. (2019). Channel-wise attention modelbased fire and rating level detection in video. CAAI Transactions on Intelligence Technology, 4(2), 117-121.

Yang, Q. F., Hwang, G. J., \& Sung, H. Y. (2020). Trends and research issues of mobile learning studies in physical education: A review of academic journal publications. Interactive Learning Environments, 28(4), 419-437.

Zafar, A., \& Hasan, H. S. (2014). Towards contextual mobile learning. International Journal of Modern Education and Computer Science (IJMECS), 6(12), 20-25.

Zhu, C., Yan, W., Cai, X., Liu, S., Li, T. H., \& Li, G. (2020). Neural saliency algorithm guide bi-directional visual perception style transfer. CAAI Transactions on Intelligence Technology, 5(1), 1-8.

Publisher's Note Springer Nature remains neutral with regard to jurisdictional claims in published maps and institutional affiliations. 

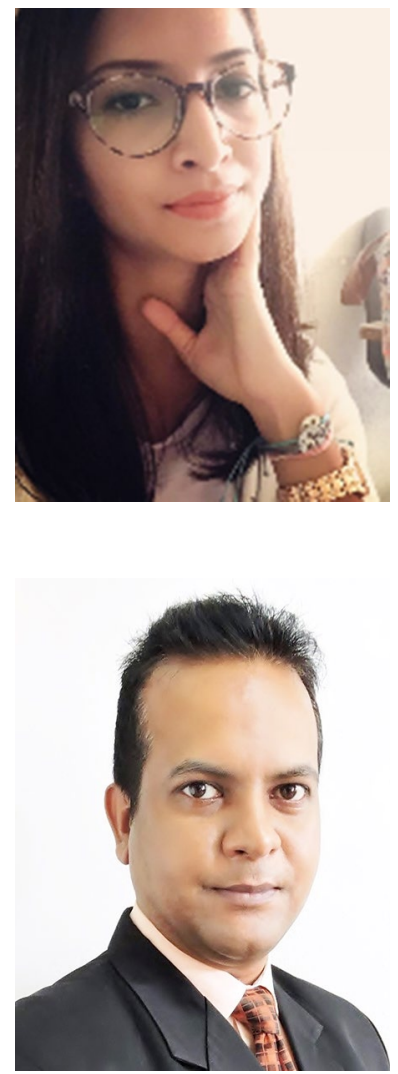

Brita Curum Ramguttee is a $\mathrm{PhD}$ candidate in the Department of Digital Technologies at the University of Mauritius. She has a diploma with distinction and a degree in Web \& Multimedia Development (Top-Up Programme) with second class first division honours from the University of Mauritius which she completed in year 2011 and 2013, respectively. She was awarded a postgraduate scholarship for being the best final year student. She is Lead Web Developer by profession; her areas of interest are mobile learning, learning analytics, context-awareness and cognitive technologies.

Dr Kavi Kumar Khedo is an associate professor in the Department of Digital Technologies at the University of Mauritius. He has a B.Eng (Hons) Computer Science degree with first class honours and $\mathrm{PhD}$ in Computer Science from the University of Mauritius. $\mathrm{He}$ has been granted over 100,000 USD as research funding and was awarded UoM Research Excellence Award 2010 and ICT Personality of the Year 2013. He has published over 50 research papers on mobile computing, ubiquitous computing and wireless sensor networks. He has published research papers in renowned international journals and has presented his research findings in reputed international conferences. He has also served on several editorial boards of international journals and TPC of international conferences such as IEEE Sensors, WILEY International Journal on Communication Systems, International Journal of Sensor Networks, IEEE Africon 2013 and IEEE ICIT 2013. 